\title{
Some Distribution of the Discrete Time Insurance Risk Model under Interest Rates with Autoregressive Structure of Order 2
}

\author{
Chunping LI \\ Department of Mathematics, Hubei Engineering University \\ Hubei Xiaogan, 432100, China \\ lichunping315@163.com
}

\begin{abstract}
-this paper discusses ruin problems in the discrete time insurance risk model under the assumption that the rate of interest is dependent upon the second autoregressive structure. By using inductive method, the recursive expressions of the distribution of the deficit at ruin, the distribution of maximum surplus before the ruin and the time that the surplus process reaches a given level $x$ for the first time are obtained, then the corresponding integral equations for the distributions are obtained.
\end{abstract}

Keywords-risk model; autoregressive structure; interest force; ruin deficit; maximum surplus

\section{INTRODUCTION}

In 1986, Ref. [1] has discussed the discrete time insurance risk model. Suppose the premiums in the unit time is constant and the claims for each period are i.i.d. in Ref [1]. Yang [2] and Sun [3] have considered some distributions about a generalized classical risk model when interest rates are identical constant. Cai [4] has discussed many distributions of the generalized classical risk model when interest rates are i.i.d.. Cai [5], Kun [6] and Hao [7] have discussed ruin probability, the deficit at ruin about the generalized classical risk model under interest rates with a dependent autoregressive structure of order 1. Su [8] has considered the ruin probability of upper bound the discrete time insurance risk model under the assumption that the rate of interest is dependent upon the second autoregressive structure.

In this paper, we discussed the discrete time insurance risk model under interest rates with autoregressive structure of order 2, by inductive technique, the recursive expressions of the distribution of ruin deficit, the maximum surplus before the ruin and the time that the surplus process reaches a given level $x$ for the first time are obtained, then the integral equation for these distributions are obtained too.

The paper is organized as follows: we definition of the model in section II ; then, in section III, IV, V, we discuss the distribution of the deficit at ruin, the maximum surplus before the ruin and the time that the surplus process reaches a given level $x$ for the first time, the recursive expressions and integral equation for the distributions are obtained; finally conclude is in section VI.

\section{DEFINITION OF THE MODEL}

We consider a discrete time risk model under interest rates with autoregressive structure of order 2 as follows

$$
U_{n}=u \prod_{k=1}^{n}\left(1+r_{k}\right)+\sum_{k=1}^{n}\left[\left(X_{k}\left(1+r_{k}\right)-Y_{k}\right) \prod_{i=k+1}^{n}\left(1+r_{i}\right)\right]
$$

where $U_{n}$ is the insurance company's surplus at time n. $U_{0}=u$ is initial reserve, $Y_{k}, r_{k}$ respectively denotes the amount of claim and rate of interest from time $k-1$ to time $k, X_{k}$ is the premiums in the time interval $[k-1, k)$. Suppose $\left\{X_{k}\right\}$ and $\left\{Y_{k}\right\}$ are i.i.d nonnegative random variables and have common distribution function $F_{X}(x)=P\left\{X_{1} \leq x\right\}$ and $F_{Y}(y)=P\left\{Y_{1} \leq y\right\}$ respectively. $\left\{r_{k}\right\}$ is the nonnegative random variables with autoregressive structure of order 2 , and $r_{k}$ satisfies

$$
r_{k}=a r_{k-1}+\beta r_{k-2}+W_{k}, k=1,2, \cdots
$$

where $0 \leq \beta \leq a<1$, which implies that the rates of interest depend heavily on the recent rates, $r_{0}>0$, $r_{-1}>0$ is constant, $\left\{W_{k}\right\}$ is i.i.d nonnegative random variables with common distribution function $F_{W}(w)=P\left\{W_{1} \leq w\right\} \quad$ and $E\left[W_{k}\right]<+\infty$. Suppose $\left\{W_{k}\right\},\left\{X_{k}\right\}$ and $\left\{Y_{k}\right\}$ are independent.

Let

and

$$
h=\alpha r_{0}+\beta r_{-1}+w
$$

$$
M_{k}=Y_{k}-\left(1+\alpha r_{k-1}+\beta r_{k-2}+W_{k}\right) X_{k}
$$

And assume $M_{1}$ has distribution function

$$
\begin{aligned}
& G\left(u, r_{0}, r_{-1}, t\right)=P\left\{M_{1} \leq t\right\} \\
& =\int_{0}^{+\infty} \int_{0}^{+\infty} F_{Y}(t+(1+h) x) d F_{X}(x) d F_{W}(w)
\end{aligned}
$$


We suppose $E\left[M_{k}\right]<+\infty, E\left[Y_{k}\right]<E\left[X_{k}\right]<+\infty$, providing a positive safety loading factor. From the above assumption, the surplus process (1) becomes the model (3

$$
U_{n}=u \prod_{k=1}^{n}\left(1+\mathrm{r}_{k}\right)-\prod_{k=1}^{n}\left(1+r_{k}\right) S_{n}=\prod_{k=1}^{n}\left(1+r_{k}\right)\left(u-S_{n}\right)
$$

where

$$
S_{n}=\sum_{k=1}^{n} M_{k} / \prod_{i=1}^{k}\left(1+r_{k}\right), S_{0}=0
$$

and

$$
\prod_{k=m}^{n}\left(1+r_{k}\right)=1 \text { if } m>n .
$$

Let $T$ is the ruin time of the insurance company, then

$$
T=\inf \left\{n: n>0, U_{n}<0\right\}=\inf \left\{n: n>0, S_{n}>u\right\}
$$

Obviously, $T$ is a stopping time.

\section{THE DEFICIT DISTRIBUTION AT RUIN}

For $x>0$, we denote the distribution of deficit at ruin with the initial reserve $u$ by

$$
G\left(u, x, r_{0}, r_{-1}\right)=P\left\{U_{T}>-x, T<\infty / U_{0}=u\right\}, x>0
$$

We get the following theorem 1 and theorem 2 about the distribution.

Theorem 1 Let $G\left(u, x, r_{0}, r_{-1}\right)$ be defined as (5), then we have

$$
G\left(u, x, r_{0}, r_{-1}\right)=\sum_{n=1}^{\infty} g_{n}\left(u, x, r_{0}, r_{-1}\right)
$$

when $n=1, g_{1}\left(u, x, r_{0}, r_{-1}\right)=$

$$
\int_{0}^{\infty}\left[G\left(u, r_{0}, r_{-1}, u(1+h)+x\right)-G\left(u, r_{0}, r_{-1}, u(1+h)\right)\right] d F_{W}(w)
$$

when $n \geq 2, g_{n}\left(u, x, r_{0}, r_{-1}\right)=$

$$
\int_{0}^{\infty} \int_{-\infty}^{u(1+h)} g_{n-1}\left(u(1+h)-t, x, h, r_{0}\right) d G\left(u, r_{0}, r_{-1}, t\right) d F_{W}(w)
$$

\section{Proof}

$G\left(u, x, r_{0}, r_{-1}\right)=P\left\{U_{T}>-x, T<\infty / U_{0}=u\right\}$

$=\sum_{n=1}^{\infty} P\left\{U_{T}>-x, T=n\right\}$

$=\sum_{n=1}^{\infty} P\left\{0 \leq U_{1}, 0 \leq U_{2}, \cdots, 0 \leq U_{n-1},-x<U_{n}<0\right\}$

$=\sum_{n=1}^{\infty} P\left\{u<S_{n}<u-\frac{x}{\prod_{k=1}^{n}\left(1+r_{k}\right)}, S_{n-1} \leq u, S_{n-2} \leq u, \cdots, S_{1} \leq u\right\}$

$=\sum_{n=1}^{\infty} g_{n}\left(u, x, r_{0}, r_{-1}\right)$

where $g_{n}\left(u, x, r_{0}, r_{-1}\right)$ is the distribution deficit at ruin time $n$.

According to definition (5), when $n=1$

$$
g_{1}\left(u, x, r_{0}, r_{-1}\right)=P\left\{-x<U_{1}<0\right\}
$$

$=P\left\{u<S_{1}<u+\frac{x}{1+r_{1}}\right\}$

$=P\left\{u\left(1+r_{1}\right)<M_{1}<u\left(1+r_{1}\right)+x\right\}$

$=\int_{0}^{+\infty} P\left\{u(1+h)<M_{1}<u(1+h)+x\right\} d F_{W}(w)$

$=\int_{0}^{\infty}\left[G\left(u, r_{0}, r_{-1}, u(1+h)+x\right)-G\left(u, r_{0}, r_{-1}, u(1+h)\right)\right] d F_{W}(w)$

when $n=2$

$$
\begin{aligned}
& g_{2}\left(u, x, r_{0}, r_{-1}\right)=P\left\{S_{1} \leq u, u<S_{2}<u+\frac{x}{\left(1+r_{1}\right)\left(1+r_{2}\right)}\right\} \\
& =P\left\{M_{1} \leq u\left(1+\alpha r_{0}+\beta r_{-1}+W_{1}\right),\right. \\
& \left.u\left(1+r_{1}\right)-M_{1}<\frac{M_{2}}{1+r_{2}}<u\left(1+r_{1}\right)-M_{1}+\frac{x}{1+r_{2}}\right\} \\
& =\int_{0}^{+\infty} \int_{-\infty}^{u(1+h)} P\left\{u(1+h)-t<\frac{M_{2}}{1+r_{2}}<\right. \\
& \left.\quad u(1+h)-t+\frac{x}{1+r_{2}}\right\} d G\left(u, r_{0}, r_{-1}, t\right) d F_{W}(w) \\
& =\int_{0}^{\infty} \int_{-\infty}^{u(1+h)} g_{1}\left(u(1+h)-t, x, h, r_{0}\right) d G\left(u, r_{0}, r_{-1}, t\right) d F_{W}(w)
\end{aligned}
$$

By inductive assumption, when $n \geq 3$, we have

$g_{n}\left(u, x, r_{0}, r_{-1}\right)=$

$\int_{0}^{\infty} \int_{-\infty}^{u(1+h)} g_{n-1}\left(u(1+h)-t, x, h, r_{0}\right) d G\left(u, r_{0}, r_{-1}, t\right) d F_{W}(w)$

Obviously $\sum_{n=1}^{\infty} g_{n}\left(u, x, r_{0}, r_{-1}\right)$ is convergence, that $G\left(u, x, r_{0}, r_{-1}\right)$ has the following theorem 2

Theorem 2 Let $G\left(u, x, r_{0}, r_{-1}\right)$ be defined as (5), then $G\left(u, x, r_{0}, r_{-1}\right)$ satisfies the following integral equation

$G\left(u, x, r_{0}, r_{-1}\right)=g_{1}\left(u, x, r_{0}, r_{-1}\right)+$ $\int_{0}^{\infty} \int_{-\infty}^{u(1+h)} G\left(u(1+h)-t, x, h, r_{0}\right) d G\left(u, r_{0}, r_{-1}, t\right) d F_{W}(w)$

\section{Proof}

$G\left(u, x, r_{0}, r_{-1}\right)=\sum_{n=1}^{\infty} g_{n}\left(u, x, r_{0}, r_{-1}\right)$

$=g_{1}\left(u, x, r_{0}, r_{-1}\right)+\sum_{n=2}^{\infty} g_{n}\left(u, x, r_{0}, r_{-1}\right)$

$=g_{1}\left(u, x, r_{0}, r_{-1}\right)+$

$\int_{0}^{\infty} \int_{-\infty}^{u(1+h)} G\left(u(1+h)-t, x, h, r_{0}\right) d G\left(u, r_{0}, r_{-1}, t\right) d F_{W}(w)$

IV. THE DISTRIBUTION OF MAXIMUM SURPLUS BEFORE THE RUIN

Denote the distribution of maximum before the ruin with the initial reserve $u$ by

$H\left(u, x, r_{0}, r_{-1}\right)=P\left\{\sup _{0 \leq k<T} U_{k} \leq x, T<\infty / U_{0}=u\right\}$

Theorem3 Let $H\left(u, x, r_{0}, r_{-1}\right)$ be defined as (10), then we can get 
(1) When $x<u$, we have

$$
H\left(u, x, r_{0}, r_{-1}\right)=0
$$

(2) When $x \geq u$, we have

$$
H\left(u, x, r_{0}, r_{-1}\right)=\sum_{n=1}^{\infty} h_{n}\left(u, x, r_{0}, r_{-1}\right)
$$

where $n=1$, we have $h_{1}\left(u, x, r_{0}, r_{-1}\right)=1-$

$$
\int_{0}^{\infty} G\left(u,, r_{0}, r_{-1}, u(1+h)\right) d F_{W}(w)
$$

and $n \geq 2$, we have

$$
h_{n}\left(u, x, r_{0}, r_{-1}\right)=
$$

$\int_{0}^{\infty} \int_{-\infty}^{u(1+h)} h_{n-1}\left(u(1+h)-t, x, h, r_{0}\right) d G\left(u, r_{0}, r_{-1}, t\right) d F_{W}(w)$.

Proof

(1) when $x<u$, according to definition(10), we easily get

$$
H\left(u, x, r_{0}, r_{-1}\right)=0
$$

(2) when $x \geq u$,we have

$$
\begin{aligned}
& H\left(u, x, r_{0}, r_{-1}\right)=\sum_{n=1}^{\infty} P\left\{\sup _{0 \leq k<T} U_{k} \leq x, T=n\right\} \\
& =\sum_{n=1}^{\infty} P\left\{0 \leq U_{1} \leq x, 0 \leq U_{2} \leq x, \cdots, 0 \leq U_{n-1} \leq x, U_{n}<0\right\} \\
& =\sum_{n=1}^{\infty} P\left\{u-\frac{x}{1+r_{1}} \leq S_{1} \leq u, u-\frac{x}{\left(1+r_{1}\right)\left(1+r_{2}\right)} \leq S_{2} \leq u\right. \\
& \left.\quad \cdots, u-\frac{x}{\prod_{k=1}^{n-1}\left(1+r_{k}\right)} \leq S_{n-1} \leq u, S_{n}>u\right\} \\
& =\sum_{n=1}^{\infty} h_{n}\left(u, x, r_{0}, r_{-1}\right)
\end{aligned}
$$

where $h_{n}\left(u, x, r_{0}, r_{-1}\right)$ is the distribution of maximum before the ruin time $n$.

According to definition (10), when $n=1$

$$
\begin{aligned}
& h_{1}\left(u, x, r_{0}, r_{-1}\right)=P\left\{S_{1}>u\right\}=P\left\{\frac{M_{1}}{1+r_{1}}>u\right\} \\
& =P\left\{M_{1}>u\left(1+\alpha r_{0}+\beta r_{-1}+W_{1}\right)\right\} \\
& =\int_{0}^{\infty}\left[1-P\left(M_{1} \leq u(1+h)\right)\right] d F_{W}(w) \\
& =1-\int_{0}^{\infty} G\left(u,, r_{0}, r_{-1}, u(1+h)\right) d F_{W}(w)
\end{aligned}
$$

when $n=2$

$$
\begin{aligned}
& h_{2}\left(u, x, r_{0}, r_{-1}\right)=P\left\{u-\frac{x}{1+r_{1}} \leq S_{1} \leq u, S_{2}>u\right\} \\
& =P\left\{u\left(1+r_{1}\right)-x \leq M_{1} \leq u\left(1+r_{1}\right),\right. \\
& \left.\quad \frac{M_{2}}{\left(1+r_{1}\right)\left(1+r_{2}\right)}>u-\frac{M_{1}}{1+r_{1}}\right\} \\
& =\int_{0}^{\infty} \int_{u(1+h)-x}^{u(1+h)} h_{1}\left(u(1+h)-t, x, h, r_{0}\right) d G\left(u, r_{0}, r_{-1}, t\right) d F_{W}(w) \\
& =\int_{0}^{\infty} \int_{-\infty}^{u(1+h)} h_{1}\left(u(1+h)-t, x, h, r_{0}\right) d G\left(u, r_{0}, r_{-1}, t\right) d F_{W}(w)
\end{aligned}
$$

By inductive assumption, when $n \geq 3$, we have $h_{n}\left(u, x, r_{0}, r_{-1}\right)=$ $\int_{0}^{\infty} \int_{-\infty}^{u(1+h)} h_{n-1}\left(u(1+h)-t, x, h, r_{0}\right) d G\left(u, r_{0}, r_{-1}, t\right) d F_{W}(w)$

From (11), we know that $\sum_{n=1}^{\infty} h_{n}\left(u, x, r_{0}, r_{-1}\right)$ is convergence. Then, we can get the follow theorem

Theorem4 Let $H\left(u, x, r_{0}, r_{-1}\right)$ be defined as (10), then $H\left(u, x, r_{0}, r_{-1}\right)$ satisfies the following integral equation

$H\left(u, x, r_{0}, r_{-1}\right)$

$=1-\int_{0}^{\infty} G\left(u,, r_{0}, r_{-1}, u(1+h)\right) d F_{W}(w)$

$+\int_{0}^{\infty} \int_{-\infty}^{u(1+h)} H\left(u(1+h)-t, x, h, r_{0}\right) d G\left(u, r_{0}, r_{-1}, t\right) d F_{W}(w)$

Proof

According by (10) and (13), we can get

$$
\begin{aligned}
& H\left(u, x, r_{0}, r_{-1}\right)=\sum_{n=1}^{\infty} h_{n}\left(u, x, r_{0}, r_{-1}\right) \\
& =h_{1}\left(u, x, r_{0}, r_{-1}\right)+\sum_{n=2}^{\infty} h_{n}\left(u, x, r_{0}, r_{-1}\right) \\
& =h_{1}\left(u, x, r_{0}, r_{-1}\right) \\
& +\int_{0}^{\infty} \int_{-\infty}^{u(1+h)} H\left(u(1+h)-t, x, h, r_{0}\right) d G\left(u, r_{0}, r_{-1}, t\right) d F_{W}(w) \\
& \text { V. THE TIME THAT THE SURPLUS PROCESS REACH A } \\
& \text { GIVEN LEVEL } X \text { FOR THE FIRST TIME }
\end{aligned}
$$

Denote the time that the surplus process reach a given level $x>0$ for the first time and probability by $T_{x}=\left\{\begin{array}{l}k \quad \text { if } \quad k=\inf \left\{n: U_{n} \geq x\right\} \\ \infty \quad \text { if } \quad \infty=\inf \left\{n: U_{n} \geq x\right\}\end{array}, P\left\{T_{x}=n\right\}=t_{n}(u, x)(11)\right.$

Let $\rho(u, x)=P\left\{T_{x}<\infty\right\}$, we can get the following theorem 5 .

Theorem 5 Let $T_{x}, P\left\{T_{x}=n\right\}=t_{n}(u, x)$ be defined as (11), then we have

(1) when $x<u, \rho(u, x)=0$

(2) when $x \geq u, \rho(u, x)=\sum_{n=1}^{\infty} t_{n}(u, x)$

where $n=1$, we have

$t_{1}(u, x)=\int_{0}^{\infty} G\left(u, r_{0}, r_{-1}, u(1+h)+y\right) d F_{W}(w)$,

when $n \geq 2$, we have

$$
t_{n}(u, x)=\int_{0}^{\infty} \int_{u(1+h)-t}^{\infty} t_{n-1}(u(1+h)-t, x) d G(t) d F_{W}(w)
$$

\section{Proof}

(1) when $x<u$, according to definition (11), we easily get $T_{x}=0$, then $\rho(u, x)=0$. 
(2) when $x \geq u$, according to definition (11), we have

$$
\rho(u, x)=P\left\{T_{x}<\infty\right\}=\sum_{n=1}^{\infty} t_{n}(u, x)
$$

when $n=1$, we have

$$
\begin{aligned}
& t_{1}(u, x)=P\left\{T_{x}=1\right\}=P\left\{U_{1} \geq x\right\} \\
& =P\left\{S_{1} \leq u-\frac{x}{1+r_{1}}\right\}=P\left\{\frac{M_{1}}{1+r_{1}} \leq u-\frac{x}{1+r_{1}}\right\} \\
& =\int_{0}^{+\infty} P\left\{M_{1} \leq u(1+h)-x\right\} d F_{W}(w) \\
& =\int_{0}^{\infty} G\left(u, r_{0}, r_{-1}, u(1+h)+y\right) d F_{W}(w) \\
& \text { when } n=2, \text { we have } \\
& t_{2}(u, x)=P\left\{U_{1}(u) \leq x, U{ }_{2}(u) \geq x\right\} \\
& =P\left\{S_{1} \geq u-\frac{x}{1+r_{1}}, S_{2} \leq u-\frac{x}{\left(1+r_{1}\right)\left(1+r_{2}\right)}\right\} \\
& =\int_{0}^{\infty} \int_{u(1+h)-x}^{\infty} P\left\{\frac{M_{2}}{1+r_{2}} \leq u(1+h)-t-\frac{x}{1+r_{2}}\right\} d G(t) d F_{W}(w) \\
& =\int_{0}^{\infty} \int_{u(1+h)-x}^{\infty} t_{1}(u(1+h)-t, x) d G(t) d F_{W}(w)
\end{aligned}
$$

By inductive assumption, when $n \geq 3$, we have

$t_{n}(u, x)=\int_{0}^{\infty} \int_{u(1+h)-t}^{\infty} t_{n-1}(u(1+h)-t, x) d G(t) d F_{W}(w)$

Obviously, we know $\sum_{n=1}^{\infty} d_{n}\left(u, x, y, r_{0}, r_{-1}\right)$ is convergence, that when $x \leq u, D\left(u, x, y, r_{0}, r_{-1}\right)$ has the following theorem 6

Theorem 6 Let $\rho(u, x)$ be defined as above, when $x \geq u$ then $\rho(u, x)$ satisfies the following integral equation

$$
\begin{aligned}
& \rho(u, x)=t_{1}(u, x) \\
& +\int_{0}^{\infty} \int_{u(1+h)-x}^{\infty} \rho(u(1+h)-t, x) d G(t) d F_{W}(w)
\end{aligned}
$$

where

$$
t_{1}(u, x)=\int_{0}^{\infty} G\left(u, r_{0}, r_{-1}, u(1+h)+y\right) d F_{W}(w) .
$$

\section{Proof}

$$
\begin{aligned}
& \rho(u, x)=\sum_{n=1}^{\infty} t_{n}(u, x)=t_{1}(u, x)+\sum_{n=2}^{\infty} t_{n}(u, x) \\
& =t_{1}(u, x)+\sum_{n=2}^{\infty} \int_{0}^{\infty} \int_{u(1+h)-x}^{\infty} t_{n-1}(u(1+h)-t, x) d G(t) d F_{W}(w) \\
& =t_{1}(u, x)+\int_{0}^{\infty} \int_{u(1+h)-x}^{\infty} \rho(u(1+h)-t, x) d G(t) d F_{W}(w)
\end{aligned}
$$

where

$$
t_{1}(u, x)=\int_{0}^{\infty} G\left(u, r_{0}, r_{-1}, u(1+h)+y\right) d F_{W}(w)
$$

\section{CONCLUSION}

In this paper, we have studied the discrete time risk model under interest rates with autoregressive structure of order 2. Via inductive method technique, some important distributions are obtained. Main results are:

1. The recursive expression of the distribution of the deficit at ruin is obtained, and its corresponding integral equation for the distribution is obtained.

2. The distribution of maximum surplus before the ruin is obtained, and its corresponding integral equation for the distribution is obtained.

3 . The time that the surplus process reaches a given level $x$ for the first time is obtained, and its corresponding integral equation for the distribution is obtained.

\section{ACKNOWLEDGMENT}

The authors are most grateful to the anonymous referees for helpful suggestions, and this paper was supported by the Youth Foundation of Hubei Educational Commission (No. Q20122603).

\section{REFERENCES}

[1] N.L. Bowers, H.U. Gerber, J.C. Hickman, D.A. Jones, C.J. Nesbitt. Actuarial Mathematics, Society of Actuaries, Itasca, IL., 1986.

[2] H. Yang, "Non-exponential bounds for ruin probability with interest effect included", Scandinavian Actuarial Journal, 1988, pp:66-79.

[3] L. J. Sun, L. Gu, L. X. Liu, "Ruin problems for the discrete time risk model", Mathematics In Economics, 2001, pp:1-9.

[4] J. Cai, "Discrete time risk model under rates of interest", Pro.Eng.Inf.Sci.2002, pp:309-324.

[5] J. Cai, "Ruin Probabilities With Dependent Rates of Interest", J.Appl.Prob.2002, pp: 312-323.

[6] F. C. Kong, L. Yu, "Ruin problems for the discrete time insurance risk model with dependent rates", Applied Mathematics a Journal of Chinese unversities, 2005, pp:320-326.

[7] H. B. Hao, J. X. Hu, C. P. Li, "A joint Distribution for the Discrete Time Insurance risk Model With Dependent Rates", Journal of Xiaogan University, 2007, pp:34-36.

[8] J. X. Su, X. J. Zhao, X. H. Li, "Ruin probability under interest rates with autoregressive structure order 2", Journal of Lanzhou University, 2004, pp:1- 4.

[9] C. P. Li, H. B. Hao, "A extreme value distribution on a renewal risk model with interest force", mathematics in economics, 2007, pp: 121-124.

[10] H. L. Yang, L. H. Zhang, "On the distribution of surplus immediately after ruin under interest force", Insurance: Statistics and probability letters, 2001, pp:329-338.

[11] B. Sundt, J. L. Tevgels, "The adjustment function in ruin estimates under interest force"'Insurance: Mathematics and Economics, 1997, pp:85-94. 